Proc. Indian Acad. Sci. (Chem. Sci.), Vol. 90, Number 3, June 1981, pp. 229-235.

(C) Printed in India.

\title{
Dielectric studies of As-Se glasses $\dagger$
}

\author{
R MOHAN and $\mathrm{K} J$ RAO* \\ Solid State and Structural Chemistry Unit, Indian Institute of Science, \\ Bangalore 560012 , India
}

MS received 7 January 1981

\begin{abstract}
Dielectric constants and loss tangents of As-Se glasses have been measured between $300 \mathrm{~K}$ and the respective glass transition temperatures and between $1 \mathrm{kHz}$ and $20 \mathrm{kHz}$. The variation of dielectric constants has been interpreted in terms of bath heteropolarity of bonds and average bond energies employing a chemically ordered network model. Various contributions to total molar polarizations have been estimated. Rapid rise of loss tangent in the vicinity of glass transitions has been interpreted in terms of rapid increase; of d.c. conductivity.
\end{abstract}

Keywords. As-Se glasses; dielectric constant; loss factor.

\section{Introduction}

Dielectric properties of amorphous selenium and arsenic selenide have been reported in the literature (Lakatos and Abkowitz 1971). But the agreement in the repoi ted values of dielectric constant for $\mathrm{As}_{2} \mathrm{Se}_{3}$ (Crevecoeur and de Wit 1971; Lakatos and Abkowitz 1971; Kolomiets 1964; Marianni et al 1973; Shimakawa et al 1977; Goyal and Srivastava 1978) is poor. Further, no systematic studies of the dielectric properties of As-Se glasses have been reported in the literature. We have, therefore, considered it important to examine the dielectric behaviour of As-Se glasses, both as a function of temperature and of composition at various frequencies. In this paper we report the dielectric properties of As-Se glasses and discuss the dependence of dielectric constants on composition using a chemically ordered network model. We have also examined dielectric losses in them in the light of their conductivity behaviour.

\section{Experimental}

Glasses were prepared from $5 \mathrm{~N}$ purity materials (Koch-Light, Co., U.K.) and the method of preparation has been described elsewhere (Mohan et al 1980). Specimens for dielectric measurements were prepared by cutting into rectangular

$\dagger$ Communication No. 108 from Solid State and Structural Chemistry Unit.

* To whom all correspondence should be made. 
blocks of glass $\left(8 \times 8 \times 0.7 \mathrm{~mm}^{3}\right)$, whose parallel faces were polished smooth with rouge powder. Silver paint electrodes were used and measurements of dielectric constant were done on a General Radio 1615-A Capacitance Bridge using external source of frequencies and General Radio 1232-A Null Detector; Temperature dependence of dielectric constants and losses were determined using a special cell shown in figure 1. Temperature was controlled to $\pm 0 \cdot 5^{\circ}$ using a temperature controller. The ambient humidities were generally very low and no special precautions were taken to avoid moisture in the cell. Measure. ments were performed from $300 \mathrm{~K}$ up to the respective glass transition temperatures $\left(T_{0}\right)$ and in some cases slightly beyond. The frequency range employed was 1 to $20 \mathrm{kHz}$.

\section{Results and discussion}

The measured values of $\epsilon^{\prime}$, real part of dielectric constant for various compositions are shown in figure 2. The error bars represent estimates obtained from considering uncertainties in thickness measurements, temperature measurements and average of several measurements. The variation of loss factor, $\tan \delta$, for different compositions at $1 \mathrm{kHz}$ is shown in figure 3 , as a function of temperature. In the inset of figure 3, variation of $\sigma_{\mathrm{d.c}} / \sigma_{\mathrm{sec}}$ with temperature has been shown for three compositions $\mathrm{As}_{30} \mathrm{Se}_{70}, \mathrm{As}_{40} \mathrm{Se}_{60}$ and $\mathrm{As}_{50} \mathrm{Se}_{50}$. In figures 4a and $\mathrm{b}$, variation of $\epsilon^{\prime}$ and $\tan \delta$ corresponding to $1 \mathrm{kHz}$ and $298 \mathrm{~K}$ are given as a function of composition. In figure 5, variation of $\tan \delta$ as a function of frequency for three compositions $\mathrm{As}_{10} \mathrm{Se}_{90}, \mathrm{As}_{40} \mathrm{Se}_{60}$ and $\mathrm{As}_{60} \mathrm{Se}_{40}$ are shown.

Figure 2 indicates that between room temperature and their respective $T_{0}^{\prime} s, \epsilon^{\prime}$ of these glasses are essentially independent of temperature. Figure 4a shows

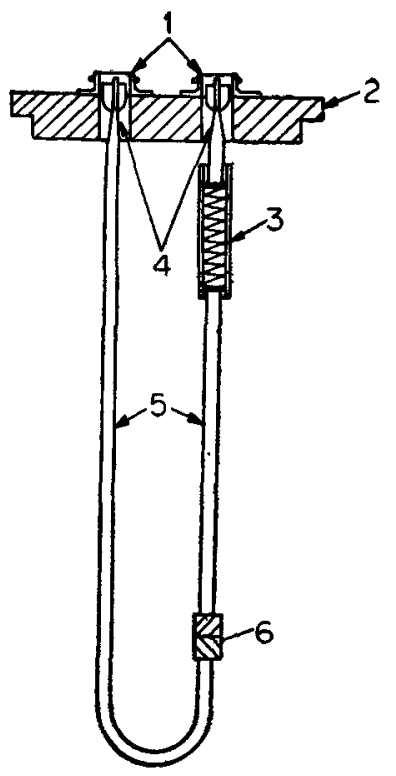

1. For BNC connectors

2. Aluminium flange

3. Spring

4. Teflon

5. Stainless steel rod and

6. Brass electrodes

Figure 1. Schematic diagram of the cell for capacitance measurement. 


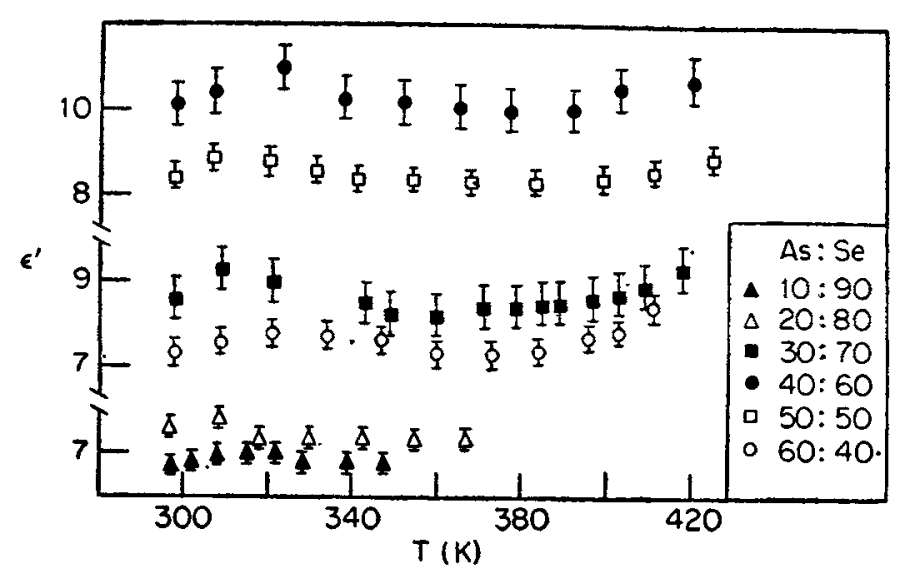

Figure 2. Variation 'of $\epsilon^{\prime}$ with temperature for various compositions at $1 \mathrm{kHz}$.

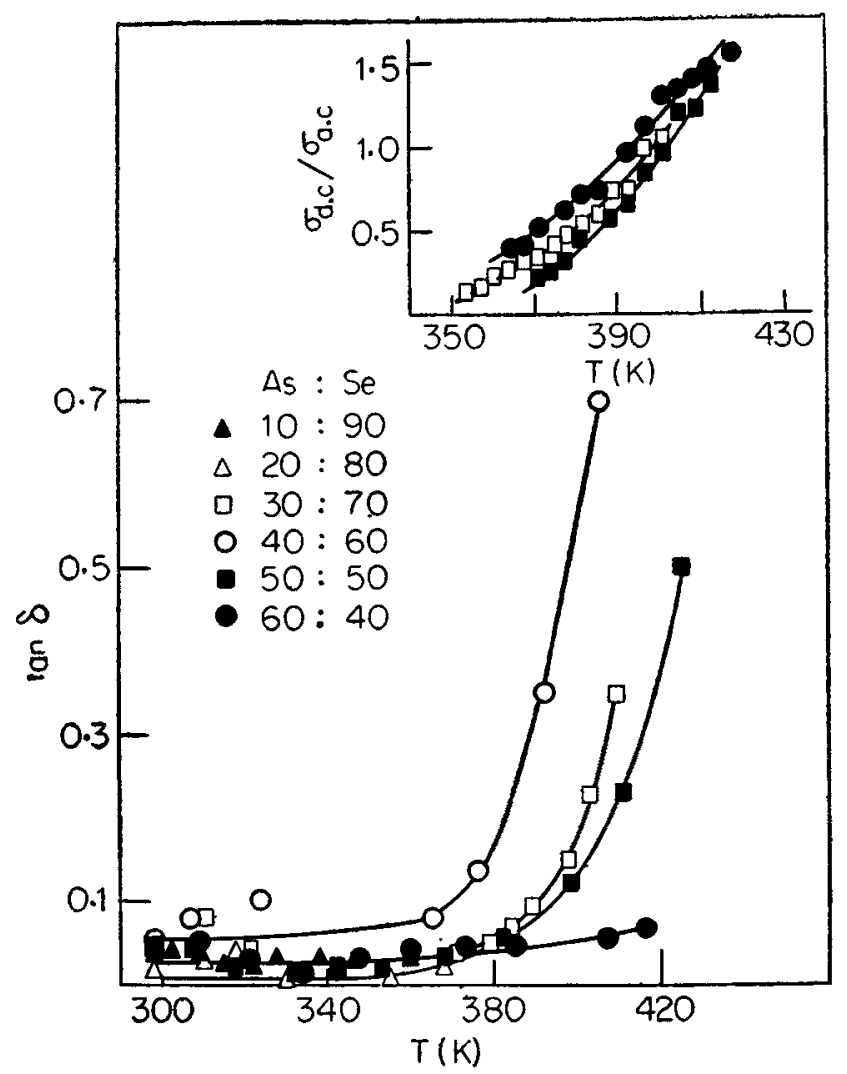

Figure 3. Variation of $\tan \delta$ with temperature at $1 \mathrm{kHz}$. In the inset, variation $\sigma_{\mathrm{d} . \mathrm{c}} / \sigma_{\mathrm{a}, \mathrm{e}}$ with temperature has been shown for three compositions; $O, \mathrm{As}_{40} \mathrm{Se}_{\mathrm{en}}$; $\square \mathrm{As}_{50} \mathrm{Se}_{70} ;$ and $\mathbf{E}, \mathrm{As}_{50} \mathrm{Se}_{50}$. 


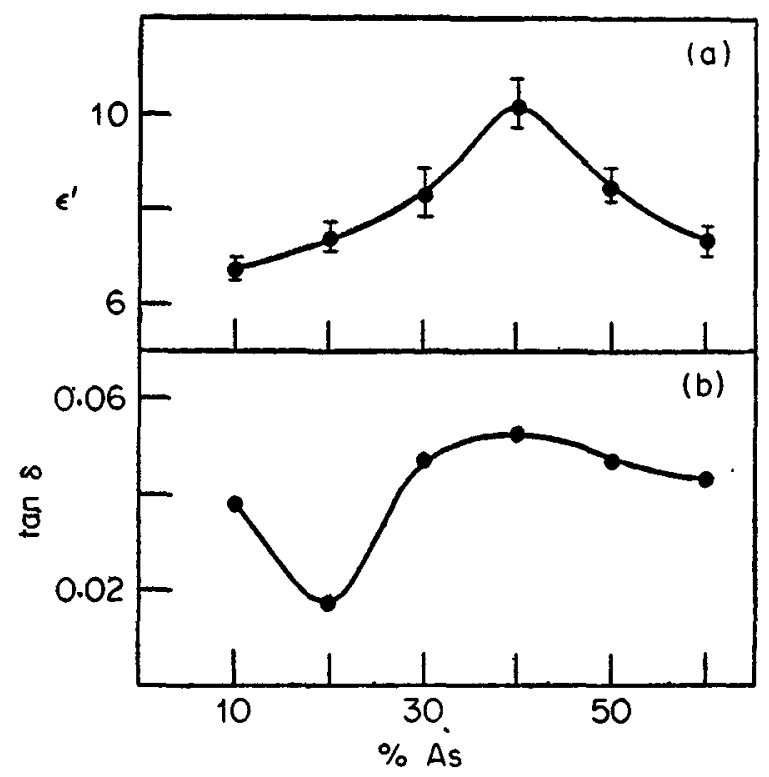

Figure 4. Variation of $\epsilon^{\prime}$ (a) and $\tan \delta$ (b) with composition at $1 \mathrm{kHz}$ and $300 \mathrm{~K}$.

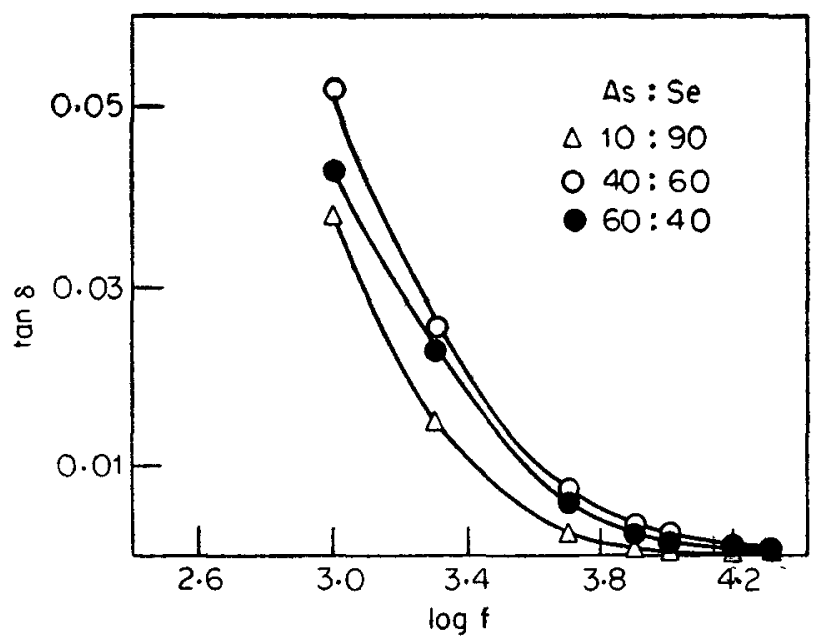

Figure 5. Variation of $\tan \delta$ with logarithmic frequency for three compositions.

that $\mathrm{As}_{40} \mathrm{Se}_{60}$ which corresponds to stoichiometric composition, has the maximum dielectric constant of $10 \cdot 3 \pm 0 \cdot 5$. This values is midway between those reported by Kolomiets (1964) and Lakatos and Abkowitz (1971) and is in good agreement with that reported by Marianni et al (1973). The extrapolated values of $\epsilon^{\prime}$ for $\mathrm{Se}$ is in good agreement with the literature value of $6 \cdot 3 \pm 0 \cdot 3$ at this frequency (1 kHz) (Lakatos and Abkowitz 1971).

One possible origin of the large values of dielectric constant and hence of polarization is the dipolar nature of As-Se bonds. However, dielectric constants may be expected to depend upon the bond concentration (number of bonds per $\mathrm{cm}^{3}$ ) 
and the bond energies (since the latter determines the polarizabilities of bonded electrons) also. The dipolar contributions may be estimated from $\Delta \chi$ values where $\Delta \chi=\left(\chi_{\mathrm{s}_{\mathrm{e}}}-\chi_{\mathrm{As}}\right)$, and $\chi$ 's are the electronegativities of the atoms. Further, we know from our earlier studies (Mohan et al 1981; Rao et al 1981) that these glasses possess chemically-ordered network and therefore, we can estimate the number of bonds which involvo $\Delta \chi$. The variation of dielectric constant $c^{\prime}$, may therefore be represented as

$$
\epsilon^{\prime} \propto[\Delta \chi \cdot f+C(1-f)],
$$

where $\mathbf{C}$ is used as an adjustable parameter. The variation of right side of expression (1) with $C=0.1$ is shown in figure 6 a (as a line on a scale indicated on the right ordinate). The measured dielectric constants $\epsilon^{\prime}$ are shown as circles on a scale corresponding to left ordinate.

Employing bond concentration and bond energy values, dependence of dielectric constants may be estimated as follows. The polarization may be assured to bel directly proportional to bond densities (number of bonds $/ \mathrm{cm}^{3}$ ) and inversey proportional to the bond energies or in other words, inversely proportional to

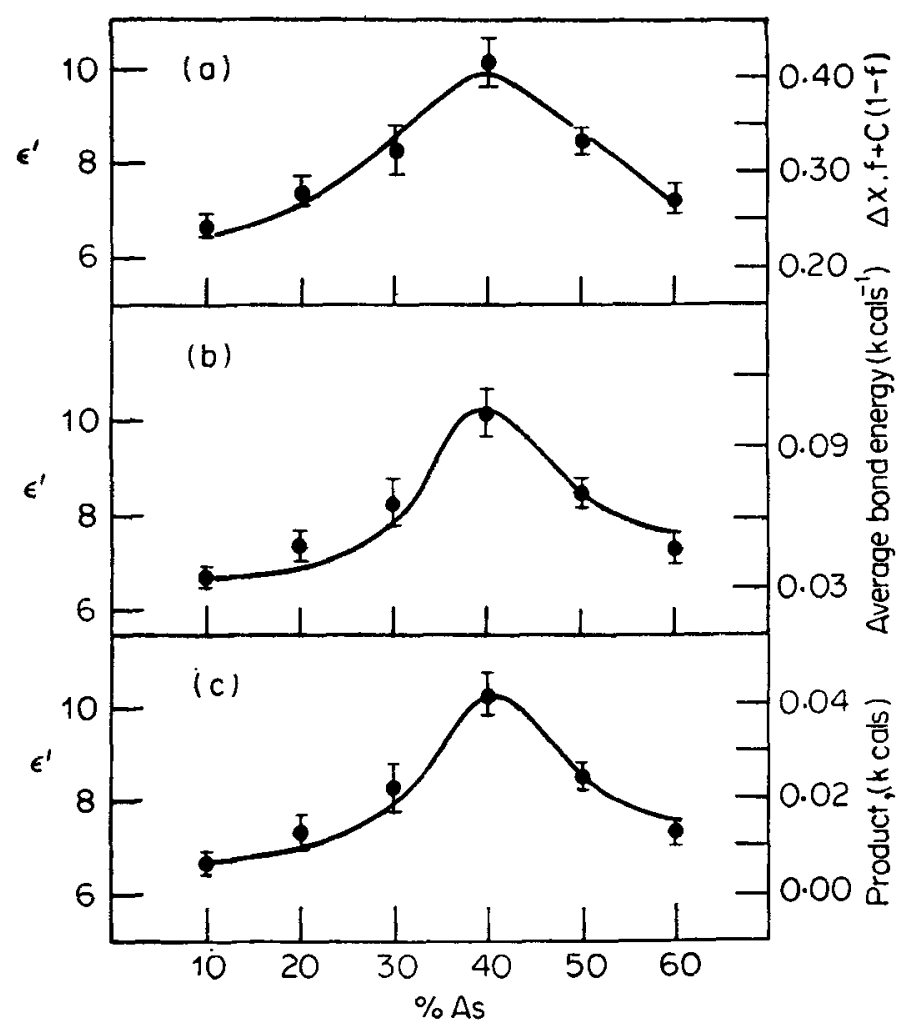

Figure 6. (a) Variation of $[\Delta \chi \cdot f+C(1-f)]$ with composition is shown as a line along with $\epsilon^{\prime}$ values (closed circles) (see text). (b). Variation of reciprocal of bond energies with composition is shown as a line along with $\epsilon^{\prime}$ values (closed circle) (see text). (c) Variation of the product, $[\{\Delta \chi \cdot f+C(1-f)\}$ (av. bond energy $\left.)^{-1}\right]$ with composition is shown as a line along with $\epsilon^{\prime}$ values (closed circles) (see text). 
the average bond energies of these glasses. Average bond energies are evaluated assuming chemical ordering in these glasses; average bond energy of $\mathrm{As}_{0} \mathrm{Se}_{y}$ is equal to average bond energy per mole of $\mathrm{As}_{z} \mathrm{Se}_{y}$ (Rao and Mohan 1980; Mohan et al 1981) divided by the number of bonds in $\mathrm{As}_{\star} \mathrm{Se}_{y}$ par mol. In figure $6 \mathrm{~b}$, the variation of reciprocal of the average bond energies are shown (as a line on a scale indicated on the right ordinate) and dielectric constants are indicated by circles. In figure $6 \mathrm{c}$ the behaviour of the product of (i) inverse of average bond energy (from figure 6b) and (ii) the r.h.s. of expression (1) (from figure 6a) is shown as a line (scale; right ordinate) along with measured dielectric constants (circles). This heuristic analysis seems to suggest that the dielectric constants depend both on the average bond energies and the polarities of bonds.

Dielectric constants can be utilised directly to evaluate molar polarization $P_{t}$ in the audiofrequency range from the Clausius-Mossotti equation. Molar polarization in the optical frequency range $P_{0}$ is similarly evaluated using the Lorentz-Lorentz equation. The polarization, $P_{t}$ and $P_{\text {, so }}$ setermined represents the total polarization and electronic polarization respectively. The atomic polarization $P_{a}$ is then easily determined by the relation $P_{a}=\left(P_{t}-P_{o}\right)$. Electronic polarization has been evaluated using the refractive index values reported by Nang et al (1979). Values of $P_{a}$ and $P_{0}$ have been listed in table 1 for the various compositions. $P_{a}$ includes contributions arising from ionic polarization of the medium also. If we assume that arsenio and selenium carry an effective charge Ze*, we can employ Szigetti (1950) relations to approximately evaluate the ionic contribution to polarization. The Szigetti relations may be combined to yield

$$
\frac{\epsilon_{0}-\epsilon_{\infty}}{\left(\epsilon_{\infty}+2\right)\left(\epsilon_{0}+2\right)} \frac{9 V}{8 \pi}=\frac{Z^{2} e^{* 2}}{p}
$$

where $\epsilon_{0}=\epsilon^{\prime}$ and $\epsilon_{\infty}=n^{2}$ and $n$ is the refractive index. This quantity has units of polarization and arises from a consideration of vibrations of ions with effective charge $\mathrm{Ze}^{*}$ only. We may, therefore, consider this as the ionic polarization $P_{b}$, of these glasses; $P_{i}$ values are listed in table 1 . it is interesting to note that $P_{t}$ is approximately $10-12 \%$ of $P_{a}$.

The loss factors $(\tan \delta$ ) exhibit an increase towards lower frequencies as shown in figure 5. The three glass compositions chosen in figure 5 , represents stoichio-

Table 1. Molar polarizations of As-Se glasses

\begin{tabular}{|c|c|c|c|}
\hline Composition & $P$ & $P_{a}$ & $P_{i}=e^{* 2} Z^{2} / P$ \\
\hline $\mathrm{As}_{10} \mathrm{Se}_{90}$ & $14 \cdot 6217$ & $0 \cdot 1754$ & $0 \cdot 0212$ \\
\hline $\mathrm{As}_{20} \mathrm{Se}_{80}$ & $19 \cdot 4533$ & $0 \cdot 4884$ & 0.0577 \\
\hline $\mathrm{As}_{30} \mathrm{Se}_{70}$ & $28 \cdot 9121$ & $1 \cdot 8086$ & $0 \cdot 2166$ \\
\hline $\mathrm{As}_{\mathbf{6 0}} \mathrm{Se}_{\mathrm{BO}}$ & $58 \cdot 2281$ & $5 \cdot 8100$ & 0.6733 \\
\hline $\mathrm{As}_{50} \mathrm{Se}_{50}$ & $35 \cdot 3733$ & $1 \cdot 4896$ & $0 \cdot 1780$ \\
\hline $\mathrm{As}_{60} \mathrm{Se}_{40}$ & $25 \cdot 9380$ & $0 \cdot 0780$ & $0 \cdot \cos 9$ \\
\hline
\end{tabular}


metric $\left(\mathrm{As}_{40} \mathrm{Se}_{60}\right)$, arsenic excess $\left(\mathrm{As}_{60} \mathrm{Se}_{40}\right)$ and selenium excess $\left(\mathrm{As}_{10} \mathrm{Se}_{90}\right)$ glasses. But the loss dependence on temperature shown in figure 3 suggests that a small dispersion in $\tan \delta$ only corresponds to the tail of a very broad absorption peak occurring at very low frequencies. The rise in loss peak in the region of $T_{0}$ for the various compositions does not follow the same trend as $T_{0}$ 's themselves. It is likely that the rise in $\tan \delta$ represents a rapid rise in d.c. conductivity (Mansingh et $a$ l 1980). It may be seen from the inset of figure 3 that $\sigma_{\text {d.e }} / \sigma_{\text {a.e }}$ attains values of the order of unity in the region where the losses increase enormously. However, our interpretation of loss data, on the basis of structural informations is limited by the frequency range covered in these glasses.

The variation of dielectric constants as a function of composition seems to be adequately accounted for assuming a chemically ordered network model for As-Se glasses. Both the dielectric constant and losses are essentially temperature and frequency-independent almost up to glass transition temperature in the audiofrequency range.

\section{Acknowledgement}

The authors thank Professor C N R Rao for his kind encouragement. We are thankful to the Department of Science and Technology for financial assistance.

\section{References}

Crevecoeur C, and de Wit H J 1971 Solid State Commun. 9445

Goyal D R and Srivastava K K 1978 Indian J. Pure Appl. Phys. 16554

Kolomiets B T 1964 Phys. Status Solidi 7359

Lakatos A I and Abkowitz M 1971 Phys. Rev. B3 1791

Mansingh A, Tandon R P and Vaid J K 1980 Phys. Rev. B21 4829

Marianni E, Trrovcova V and Lezal D 1973 Phys. State Solidi A16 K51

Mohan R, Sudha Mahadevan and Rao K J 1980 Mater. Res. Bull. 15917

Mohan R, Panchapagesan T S and Rao K J 1981 Bull. Mater. Sci. 329

Nang T T, Okuda M and Matsushita T 1979 J. Non-Cryst. Solids 33311

Rao K J and Mohan R 1980 J. Phys. Chem. 841917

Rao K J, Mohan R, Sarode P R and Hegde M S 1981 Pramäna 16309

Shimakawa K, Nitta S and Mori M 1977 Phys. Rev. B16 4519

Szigetti B 1950 Proc. $R$. Soc. A204 51 